\title{
Non-affine response: jammed packings versus spring networks
}

\author{
Wouter G. Ellenbroek ${ }^{1,2}$, Zorana Zeravcic ${ }^{2}$, Wim van SaArloos ${ }^{2}$ and Martin van Hecke ${ }^{3}$ \\ 1 Department of Physics and Astronomy, University of Pennsylvania, Philadelphia, PA 19104-6396, USA \\ 2 Instituut-Lorentz, Universiteit Leiden, Postbus 9506, 2300 RA Leiden, The Netherlands \\ ' 3 Kamerlingh Onnes Lab, Universiteit Leiden, Postbus 9504, 2300 RA Leiden, The Netherlands
}

PACS 45.70.-n - Granular systems

PACS 46.25.-y - Static elasticity

PACS 64.60.aq - Networks

\begin{abstract}
We compare the elastic response of spring networks whose contact geometry is derived from real packings of frictionless discs, to networks obtained by randomly cutting bonds in a highly connected network derived from a well-compressed packing. We find that the shear response of packing-derived networks, and both the shear and compression response of randomly cut networks, are all similar: the elastic moduli vanish linearly near jamming, and distributions characterizing the local geometry of the response scale with distance to jamming. Compression of packing-derived networks is exceptional: the elastic modulus remains constant and the geometrical distributions do not exhibit simple scaling. We conclude that the compression response of jammed packings is anomalous, rather than the shear response.
\end{abstract}

The jamming transition governs the onset of rigidity in disordered media as diverse as foams, colloidal suspensions, granular media and glasses [1]. While jamming in general is controlled by a combination of density, shear stress and temperature, most progress has been made for ' frictionless soft spheres that interact through purely repulsive contact forces, and that are at zero temperature and zero load $[2-7]$. This simple model applies to static foams or emulsions $[8,9]$, and represents a simplified version of granular media, if one ignores friction $[10,11]$ and nontrivial grain shapes [12-15].

From a theoretical point of view, this model is ideal for two reasons. First, it exhibits a well defined jamming point, "point $J "$, which in the limit of large system sizes, occurs at a well-defined density $\phi=\phi_{c}[2]$. Here the system is a disordered packing of frictionless undeformed spheres, which is marginally stable and isostatic, i.e., its contact number (average number of contacts per particle) $z$ equals $z_{\text {iso }}=2 d$ in $d$ dimensions $[2,16]$. Second, in recent years it has been uncovered that the mechanical and geometric properties of such jammed packings exhibit a number of non-trivial power law scalings as a function of the distance to the jamming point: (1) The excess contact number $\Delta z:=z-z_{\text {iso }}$ scales as $\left(\phi-\phi_{c}\right)^{1 / 2}[2,6,9,10]$; (2) The ratio of shear $(G)$ and bulk $(K)$ elastic moduli vanishes at point $J$ as $G / K \sim \Delta z[2]$.

The latter behavior - a shear rigidity which becomes much smaller than the compression modulus as the jamming point is approached - is in many ways surprising. It also differs markedly from what is found in two simplified models of jammed systems, effective medium theory (EMT) and random elastic networks, as is illustrated schematically in fig. 1 for the simple case of harmonic particles. EMT predicts that the elastic moduli vary smoothly through the isostatic point where $\Delta z=0$ and that the moduli are of order of the local spring constant $k$. This is because effective medium theory is essentially "blind" to local packing considerations and isostaticity. Thus, besides failing to capture the vanishing of $G$ near jamming, its prediction for the bulk modulus fails spectacularly as well: it predicts finite rigidity below isostaticity.

The failure of EMT to describe elasticity near jamming motivated earlier suggestions that elasticity of jammed packings might be captured by random networks of springs - this problem is known as rigidity percolation [8,17-19]. However, in such random spring networks, both $G$ and $K$ are expected to go to zero as $k \Delta z$, as fig. 11: illustrates [17].

Thus, while from the point of view of effective medium theory the shear rigidity of jammed packings behaves anomalously, from the point of view of rigidity percolation, the compression modulus behaves unexpectedly. What sets jammed packings apart from either of these two limiting models? How to understand the difference in terms of the local packing or response? Is the difference with 

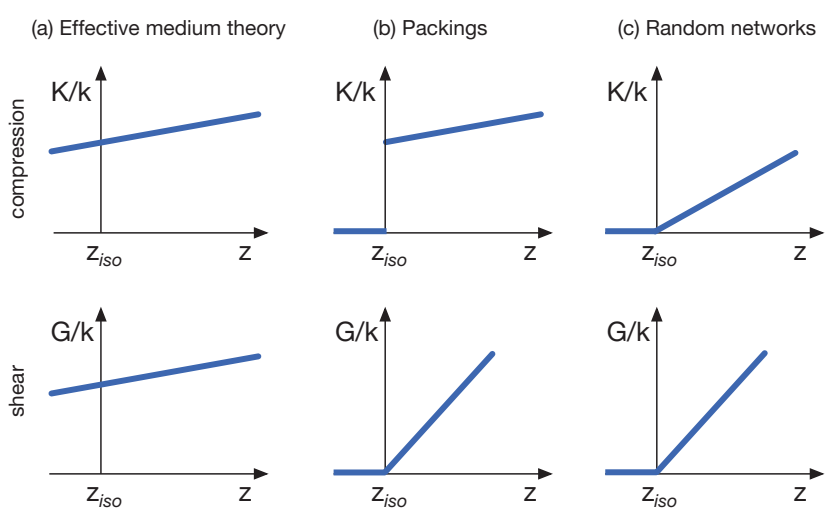

Fig. 1: Schematic comparison of the variation of shear $(G)$ and bulk $(K)$ elastic moduli as function of distance to jamming. (a) In effective medium theory, all elastic moduli are simply of the order of the local spring constant $k$, and moreover, the theory does not account for whether the packing is rigid or not. (b) In jammed packings of harmonic particles, the bulk modulus $K$ remains constant down to the jamming transition, where it vanishes discontinuously, whereas the shear modulus $G$ vanishes linearly in $\Delta z$. (c) In random networks of elastic springs, both elastic moduli vanish linearly with $\Delta z$.

rigidity percolation visible in the scaling behavior of the response of packings? These are issues we aim to clarify in this paper. Our approach will hinge on characterizing the elastic response at the level of the bonds. After all, the elastic moduli characterize changes in elastic energy $\Delta E$ under deformations, and $\Delta E$ simply is a sum of the changes in elastic energy of all contacts (bonds) in the system.

By probing the nature of the local response of packingderived and randomly cut networks, we find that we can distinguish two cases. In the "generic" case, all geometrical characterizations exhibit simple scaling and the elastic moduli scale as $\Delta z$ - this describes shear and bulk deformations of randomly cut networks, as well as shear deformations of packing-derived networks. Packing derived networks under compression form the "exceptional" case: the fact that the compression modulus remains of order $k$ near jamming is reflected in the fact that various characteristics of the local displacements do not exhibit pure scaling. We connect these findings to recent theoretical work by Wyart [20,21].

Linear Response. - All numerical results presented in this paper concern quasistatic linear response of systems to global shear or compressional forcing. First we generate, for a range of pressures, ensembles of 50 twodimensional jammed packings of 1024 frictionless particles with one-sided harmonic forces $(k=1)$ using a Molecular Dynamics simulation (for details, see [22]). Our linear response calculations are based on the dynamical matrix. We decompose, for linear deformations, the relative displacement $\mathbf{u}_{i j}$ of neighboring particles $i$ and $j$ in components parallel $\left(u_{\|}\right)$and perpendicular $\left(u_{\perp}\right)$ to $\mathbf{r}_{i j}$, where

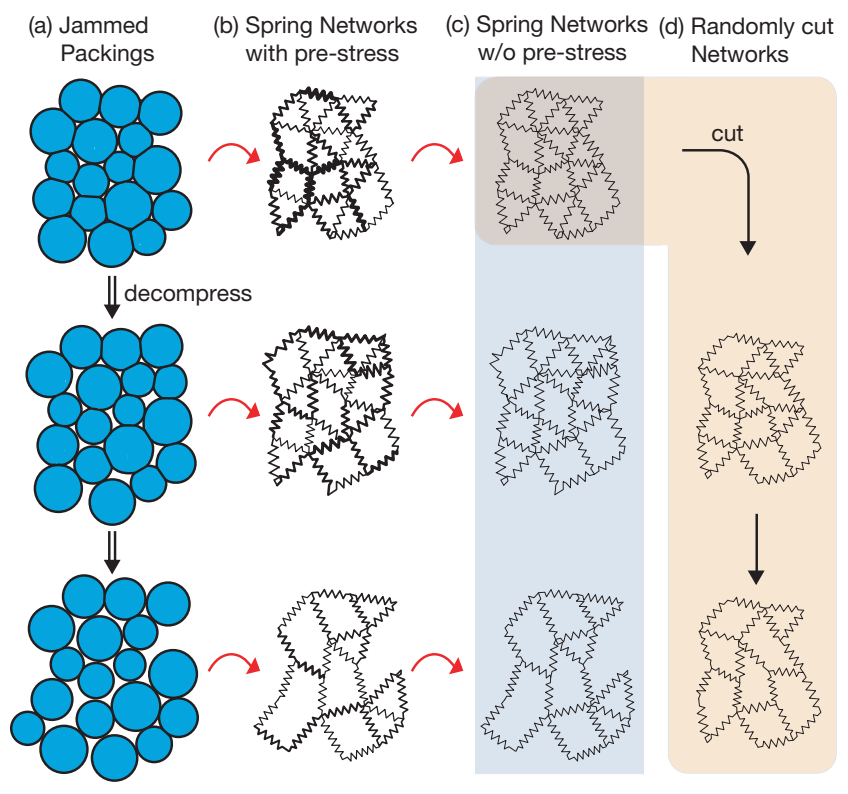

Fig. 2: Two families of spring networks - see text for details.

$\mathbf{r}_{i j}$ connects the centers of particles $i$ and $j$. In these terms the change in energy takes a simple form [5,23],

$$
\Delta E=\frac{1}{2} \sum_{i, j} \frac{k}{2}\left(u_{\|, i j}^{2}-\frac{f_{i j}}{k r_{i j}} u_{\perp, i j}^{2}\right) .
$$

The dynamical matrix $\mathcal{M}_{i j, \alpha \beta}$ is obtained by rewriting eq. (11) in terms of the independent variables, $u_{i, \alpha}$, as

$$
\Delta E=\frac{1}{2} \mathcal{M}_{i j, \alpha \beta} u_{i, \alpha} u_{j, \beta}
$$

Here $f_{i j}$ are contact forces, $k$ denotes the stiffness of the spring $1, \mathcal{M}$ is a $d N \times d N$ matrix with $N$ the number of particles, indices $\alpha, \beta$ label the coordinate axes, and the summation convention is used. The dynamical matrix contains all information on the elastic properties of the system, and in particular describes the linear response to external forcing $f_{i, \alpha}^{\text {ext }}$ as $[7,24]$ :

$$
\mathcal{M}_{i j, \alpha \beta} \quad u_{j, \beta}=f_{i, \alpha}^{\mathrm{ext}}
$$

Two Families of Spring Networks. - We start by noting that the analysis of the linear response of jammed packings of particles with one-sided harmonic interactions (fig. 2a) is exactly equivalent to that of networks of appropriately loaded harmonic springs (fig. $2 \mathrm{~b}$ ), with the nodes of the network given by the particle centers and the geometry and forces of the spring network determined by the force network of the packing.

In all that follows, we ignore the pre-stress term $\frac{f_{i j}}{k r_{i j}} u_{\perp, i j}^{2}$ which is subdominant near jamming — we have

\footnotetext{
${ }^{1}$ For our harmonic potential, $k=1$ for each contact, but the procedure works equally well for more general potentials, for which $k_{i j}$ is simply the value of the second derivative of the potential, evaluated at the initial distance $r_{i j}$.
} 
Non-affine response: jammed packings versus spring networks
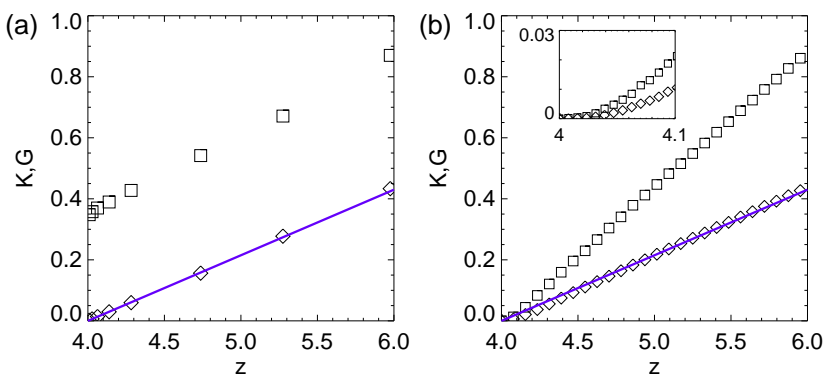

Fig. 3: Shear (diamonds) and bulk (squares) elastic moduli as function of distance to jamming for (a) jammed harmonic packings (b) randomly cut spring networks. Both panels have the same linear fit for the shear modulus (blue line). Inset: enlargement of the behavior of $K$ and $G$ near $z=4$.

checked that its exclusion does not affect the results [6]. The system without pre-stress is equivalent to a "neutral" spring network where all contacts are replaced by springs at their equilibrium length so that $f_{i j}=0$ for all contacts (fig. 2.). For such a neutral spring network the dynamical matrix becomes particularly simple, as its only non-zero elements are simply given by geometry and by the bond strengths $k$ of each bond.

We follow two routes to approach the (un)jamming transition by lowering the contact number in the neutral networks. In the first route, we map jammed packings under increasingly low pressure (fig. 2a to neutral, packingderived spring networks (fig. 2r) - the geometry of these networks and the contacts that are broken when point $\mathrm{J}$ is approached are thus set by real packings that were created using the MD protocol described in Ref. [22]. In the second route, we start from a neutral spring network that is obtained from a heavily compressed jammed packing for which $z \approx 5.98$. We then create randomly cut networks with lower contact number by randomly removing springs [25], making sure that we do not create local disconnected patches and that each node in the network remains connected by at least three springs (fig. 2 $\mathrm{d}$ ) - the geometry of these networks becomes increasingly random. Note that no relaxation is needed after removing springs because the neutral network has $f_{i j}=0$ in each contact.

Elastic Moduli. - To analyze the linear response, we impose an infinitesimal strain deformation of order $\varepsilon$, implemented by the appropriate changes in rest lengths of all bonds that cross the boundary of the periodic box of size $L \times L$. This amounts to replacing $\mathbf{u}_{i j}$ in eq. (1) by $\mathbf{u}_{i j}-$ $\mathbf{u}_{i j}^{\mathrm{b}}$, where $\mathbf{u}_{i j}^{\mathrm{b}}$ denotes the appropriate shift of magnitude $\varepsilon L$ at bonds $i j$ that cross the boundary, and is zero for interior bonds. Keeping track of this substitution in going from eq. (1) to eq. (3), these constant terms are taken to the right hand side, and thus act like an effective $f^{\text {ext }}[7]$ that is proportional to $\varepsilon$. The response of the system to this shape or volume change of the box is then calculated by solving equation (3) for this effective external force.

The moduli are extracted from the energy (eq. (2)) ac- cording to

$$
K, G=\frac{\Delta E}{2 V \varepsilon^{2}},
$$

for a uniform strain, $\varepsilon_{x x}=\varepsilon_{y y}=\varepsilon$ for compression, and $\varepsilon_{x y}=\varepsilon$ for shear. Here $V$ is the volume of the system.

In fig. 3 we show the scaling of the elastic moduli $G$ and $K$ thus obtained, as a function of the contact number $z$ for both packing-derived and randomly cut spring networks. The main point is that these two families exhibit different scaling behavior: for randomly cut networks, both moduli vanish as $\Delta z$, while for the packing-derived networks only the shear modulus $G$ goes to zero - the compression modulus $K$ remains finit 2 . The behavior of the randomly cut networks is consistent with what is expected for rigidity percolation in random spring networks $[17,18]$, while the behavior for packing-derived networks is in agreement with earlier data for jammed packings [2,7]. Hence, from the point of view of rigidity percolation, the anomaly in jammed packings is thus that the compression modulus $K / k$ stays finite as $\Delta z \rightarrow 0$.

Note that the dynamical matrix of both types of networks contains only geometric information about the network, since the spring constant $k=1$ for each bond. Hence the crucial difference between packing-derived networks and randomly cut networks that is causing the bulk modulus to be different must have a purely geometric origin.

Nonaffinity of Response. - We will now connect the scaling of the elastic moduli to the local deformation field. One tool that we use to probe the degree of nonaffinity of the response near point $J$ is $P(\alpha)$, the probability density function (PDF) of the displacement angles $\alpha_{i j}[7]$. Here $\alpha$ denotes the angle between $\mathbf{u}_{i j}$ and $\mathbf{r}_{i j}$, or,

$$
\tan \alpha_{i j}=\frac{u_{\perp, i j}}{u_{\|, i j}} .
$$

In EMT, the displacements of the particles are prescribed by an affine deformation field. Affine compression corresponds to a uniform shrinking of the bond vectors, i.e. $u_{\perp, i j}=0$ while $u_{\|, i j}=-\varepsilon r_{i j}<0$ : the corresponding $P(\alpha)$ exhibits thus a delta peak at $\alpha=\pi$. The effect of an affine shear on a bond vector depends on its orientation, and for isotropic random packings $P(\alpha)$ is flat.

The results for packing-derived networks are shown in fig. 4hac. Note that far away from jamming, the PDFs are similar to the EMT predictions: a peak at $\pi$ under compression, and a flat PDF under shear. When approaching the unjamming transition, a peak at $\alpha=\pi / 2$ develops, which signifies that an increasingly large fraction of contacting particles mostly slide past each other. However, under shear, this peak is much more pronounced than under compression, and under compression the PDF retains a significant shoulder between $\pi / 2$ and $\pi$.

\footnotetext{
${ }^{2}$ Here and in what follows, $\Delta z=z-z_{\mathrm{c}} \approx z-z_{\text {iso }}$, where for packing-derived (randomly cut) networks $z_{\mathrm{c}}=4$ (4.045). The discrepancy between $z_{\mathrm{c}}$ and $z_{\text {iso }}$ (see inset of fig. 3) for the randomly cut networks is not a finite size effect, but can be attributed to the precise cutting protocol.
} 


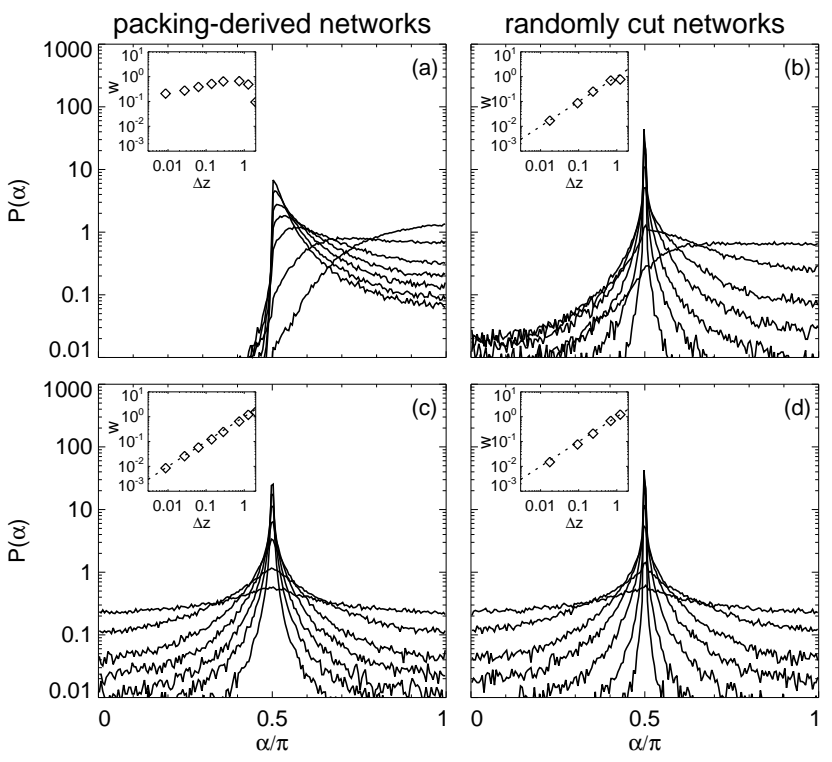

Fig. 4: The PDF of the displacement angles $P(\alpha)$ for compression $(\mathrm{a}, \mathrm{b})$ and shear $(\mathrm{c}, \mathrm{d})$. The seven curves denote, in order of decreasing peak height at $\alpha=\pi / 2$, $z=4.008,4.027,4.063,4.14,4.28,4.74,5.27$. (a,c) For packingderived networks, $P(\alpha)$ for compression and shear appear rather different. (b,d) For randomly cut networks, $P(\alpha)$ develops the same peaked structure when $z \rightarrow 4$ for compression and shear. Insets: The width of the peaks (defined as the width of the interval containing the central $50 \%$ of the data), as a function of $\Delta z$. The dotted lines indicate $w \sim \Delta z$ for all cases except compression of bead packings.

The results from the randomly cut networks are shown in fig. 4bd: a strong peak develops in $P(\alpha)$ as $\Delta z$ decreases, both for the response to shear and to compression. The relative displacements of contacting particles in response to compression thus signal an important difference between packing-derived networks and random networks.

Scaling Arguments for Non Affinity. - Wyart and coworkers have given arguments for estimating the energies and local deformations of soft (low energy) modes starting from purely floppy (zero energy) modes [5, 25]. They construct trial soft modes that are basically floppy modes, obtained by cutting bonds around a patch of size $\ell^{*}$, and then modulating these with a sine function of wavelength $\ell^{*}$ to make the displacements vanish at the locations of the cut bonds. Here $\ell^{*} \sim 1 / \Delta z$ is a characteristic length scale $[5-7,20]$. In particular, for the local

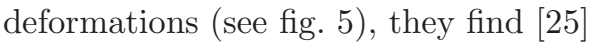

$$
\frac{u_{\|}}{u_{\perp}} \sim \frac{1}{\ell^{*}} \rightarrow \frac{u_{\|}}{u_{\perp}} \sim \Delta z,
$$

where symbols without indices $i j$ refer to typical or average values of the respective quantitie 3 . Note that the width $w$ of the peak in $P(\alpha)$ is, close to the jamming

\footnotetext{
${ }^{3}$ In earlier work [7], we have argued that the scaling $u_{\|} / u_{\perp} \sim \Delta z$ can also be understood by balancing the first and second terms in
}

(a) Floppy mode

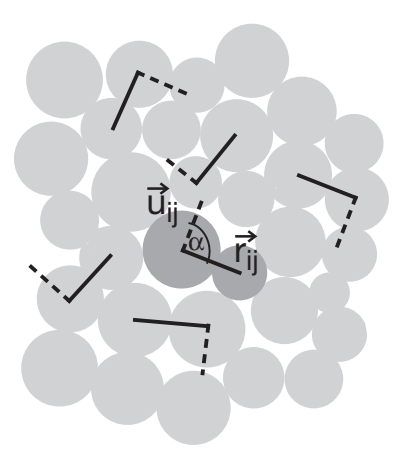

(b) Soft mode

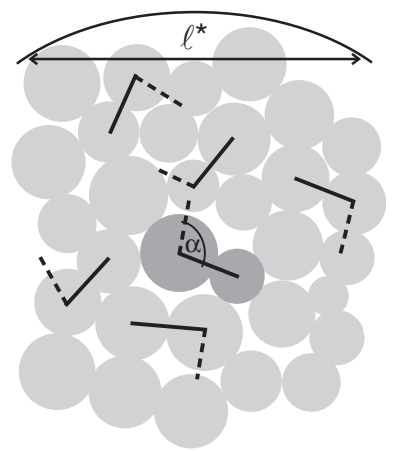

Fig. 5: Illustration of Wyart's argument [25] for $u_{\|} / u_{\perp}$ : Left: a floppy patch of material, obtained by cutting bonds on the outer edge, in which all contacts have exactly $\alpha=\pi / 2$ upon distortion, in accord with the definition of a floppy mode [23]. Right: a weakly distorted floppy mode (also called trial soft mode) can be thought of as a floppy mode that is distorted elastically on a scale $\ell^{*}$. Accordingly all angles $\alpha$ are slightly different from $\pi / 2$, as indicated in the figure. The dashed lines denote the relative displacement pairs of contacting particles, marked by the solid line connecting their centers.

transition, roughly $w \sim u_{\|} / u_{\perp}$, because $\left|\alpha_{i j}-\pi / 2\right| \approx$ $u_{\|, i j} / u_{\perp, i j}$ if $u_{\|, i j} \ll u_{\perp, i j}$.

The question is now, whether the linear response follows this prediction for the soft modes, for our two families of networks. The insets of fig. 4 show that the scaling behavior (6) is consistent with our data for the width $w$ of the peak of $P(\alpha)$ for packing-derived networks under shear, and for randomly cut networks under either compression and shear deformations. The $P(\alpha)$ for compression of packing-derived networks is the exceptional case. The peak of $P(\alpha)$ does not grow as much, and a substantial shoulder for large $\alpha$ remains even close to jamming: the tendency for particles to move towards each other remains much more prominent under compression.

Fraction of Compressed Bonds. - In order to clarify the significance of the large- $\alpha$ shoulder, let us analyze the fraction of significantly compressed bonds. Intuitively, it is clear that this fraction should be at the root of the difference between randomly cut networks, whose compression modulus $K$ vanishes near jamming, and packingderived networks whose $K$ does not. Indeed, suppose we compress a packing-derived network. When a finite fraction of the bonds gets shortened with a finite fraction of the strain $\varepsilon$, then $K$ will be proportional to the bond strength $k$ - this simply follows from the expression for the energy change $\Delta E$, eq. (10).

To quantify this, we define the fraction $\rho_{\text {comp }}$ of bonds

the energy expansion (eq. (1) which yields the scaling $u_{\|} / u_{\perp} \sim \sqrt{\delta}$, with $\delta$ is the typical overlap. For jammed packings, where the prestress term is taken into account, this result is consistent with [6] in view of the scaling $\Delta z \sim \sqrt{\delta}$. However, as we show here, even if the pre-stress term is ignored in the dynamical matrix, very similar scaling is obtained. 

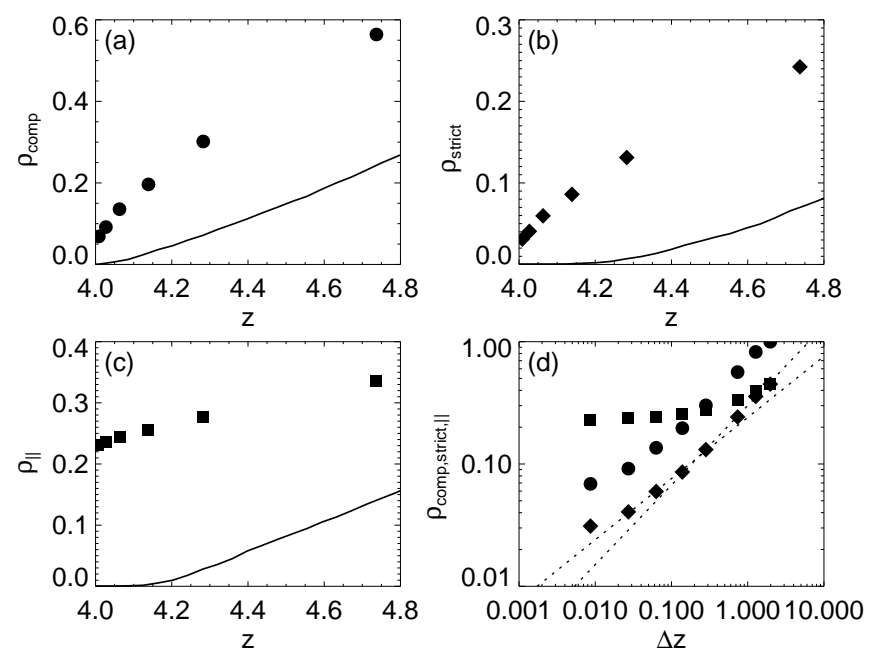

Fig. 6: Scaling of (a) $\rho_{\text {comp }}$, (b) $\rho_{\text {strict }}$, and (c) $\rho_{\|}$, as a function of $z$ for compression of packing-derived networks (symbols) and randomly cut networks (curves). (d) The packing data on a $\log$ scale, emphasizing the rapid rise of $\rho_{\text {comp }}$ at small $\Delta z$. The dotted lines mark exponents 0.5 and 0.65 , to guide the eye there is no clean scaling.

whose local response has $\alpha>3 \pi / 4$, i.e., contact pairs which upon compression move more towards each other than they move sideways $\left(u_{\|}<-\left|u_{\perp}\right|<0\right)$. If the PDF $P(\alpha)$ was governed by a single scale, the observed scaling of the width of the peak of $P(\alpha) \sim \Delta z$ in accord with ([6), suggests that $\rho_{\text {comp }} \sim \Delta z$ near jamming.

In fig. 6 we compare the scaling of $\rho_{\text {comp }}$ for both our types of networks. The $P(\alpha)$ for random networks can be described by a single scale $1 / \ell^{*} \sim w \sim \Delta z$ (fig. 目), and indeed the corresponding $\rho_{\text {comp }}$ is linear in $\Delta z$ (fig. 6 6 ). For packing-derived networks close to jamming, $\rho_{\text {comp }}$ rises more rapidly than linearly, and is much larger than for the randomly cut networks. This shows that under compression of packings a significant fraction of the contacts remains non-sliding and that single-parameter scaling does not apply - indeed, while our randomly cut networks are consistent with a linear variation of $\rho_{\text {comp }}$, if we fit our data for packing-derived networks to a power law form $\rho_{\text {comp }} \sim(\Delta z)^{\zeta}$, we do not find a clear scaling $(\zeta \approx 0.65$, but only over 1 decade in $\Delta z$ ).

In principle, many of the bonds with $\alpha>3 \pi / 4$ could have anomalously small $u_{\|}$- to check that this is not the case, we have also investigated $\rho_{\text {strict }}$, the fraction of bonds whose local response has $\alpha>3 \pi / 4$ and $u_{\|}<-\varepsilon r_{i j}$, and $\rho_{\|}$, the fraction of bonds whose local response has $u_{\|}<-\varepsilon r_{i j}$. The latter condition can be phrased as the fraction of bonds that are more compressed than they would be if the response were affine. As shown in fig. 6b$c$, these measures of compressed bond fractions are also much larger for compression of packing-derived networks.

For compression of packing-derived networks, since $K / k$ remains finite for $\Delta z \rightarrow 0$, one should expect a finite fraction of bonds with $u_{\|}$of order $\varepsilon$ - consistent with this $\rho_{\|}$ remains finite in this case. Although the $P(\alpha)$ in fig. 4 a do not appear to be governed by a single scale, a tentative argument can be given why the rise in $\rho_{\text {comp }}$ is steeper than linear for small $\Delta z$ : Assume the typical $u_{\perp}$ is still of the order $\varepsilon / \sqrt{\Delta z}$, as is the case for compression of randomly cut networks (from combining eq. (6) with eq. (1) and $K \sim k \Delta z)$. Then, the relevant scale in $P(\alpha)$ would be set by $u_{\|} / u_{\perp} \sim \sqrt{\Delta z}$, and one would find $\rho_{\text {comp }} \sim \sqrt{\Delta z}$. As expected, we do not find such a clear scaling in fig. 6 $\mathrm{d}$, but the rapid initial rise is clearly visible.

In conclusion, we find that the non-affine displacements in random spring networks and sheared jammed packings all share the same simple scalings of $P(\alpha)$, as well as having elastic moduli which scale as $k \Delta z$, where $k$ denotes the bond stiffness. The response of jammed packings to compression is the exceptional case: $P(\alpha)$ has more structure than a single peak, naive scaling breaks down and $K \sim k$.

Interpretation in terms of the space of force networks. - We finally briefly discuss these issues within the framework developed by Wyart [20,21] for the response of frictionless granular packings. For a network consisting of $N$ particles and $z N / 2$ contacts, any imposed deformation can be expressed in terms of the change of the rest lengths of some bonds in the network. After perturbing one or more bonds, for example in a way which corresponds to a global shear or compression of the packing, there will be an energy minimization involving the $d N$ degrees of freedom (displacements $\mathbf{u}_{i}$ ). Hence, the space of responses to perturbations that cost energy has dimension $z N / 2-d N=\Delta z N / 2$. An equivalent way to view this is that after perturbing the rest lengths of the bonds, the particles will move so as to satisfy the $d N$ local equations of force balance. Therefore the force response network can be expressed in a basis $\left\{\mathbf{f}^{(i)}\right\}$ of the $\Delta z N / 2$-dimensional solution space $\mathcal{F}$ of of the force balance equations.

The force space thus defined is very similar to the solution space of the force network ensemble [26-28], where one studies the space of allowed force configurations, $\mathcal{F}^{\text {fne }}$ for a given contact geometry and externally imposed pressure. Let us define the extended force network ensemble, as the ensemble of all allowed force configurations, without the constraint that the pressure be fixed [28]. This force space is precisely the $\Delta z N / 2$-dimensional space spanned by the orthonormal basis $\left\{\mathbf{f}^{(i)}\right\}$ defined above.

Now, if we fix the pressure, this leads to an additional constraint. By a simple rotation in force space it is possible to choose the $\left\{\mathbf{f}^{(i)}\right\}$ such that $\mathbf{f}^{(1)}$ precisely gives the direction of increasing pressure, so that all other base vectors are perpendicular to the pressure direction [28] the force ensemble with fixed pressure simply results from projecting out the $\mathbf{f}^{(1)}$ direction from $\mathcal{F}$.

Suppose we externally impose changes in the rest lengths of the bonds, denoted by $\mathbf{y}$ - for a compression, we may for example increase all rest lengths. Wyart [20] then shows that the energy change corresponding to such 
external forcing can be expressed as

$$
\Delta E=\frac{1}{2} \sum_{i=1}^{N \Delta z / 2}\left\langle\mathbf{f}^{(i)} \mid \mathbf{y}\right\rangle^{2},
$$

where $\langle\cdot \mid \cdot\rangle$ denotes the scalar product in force space. If we consider a deformation $\mathbf{y}$ of which the components are of order $\epsilon$ then in general we may assume that there will be no correlation between $\mathbf{y}$ and the force space $\mathcal{F}$. The dominant contribution to the squared inner product in eq. (7) is then $\sum_{m}\left(f_{m}^{(i)}\right)^{2} y_{m}^{2} \sim \sum_{m} \epsilon^{2} / N \sim \epsilon^{2}$, and summing these over all basis vectors gives $\Delta E \sim N \Delta z \epsilon^{2}$, making the energy extensive and proportional to the distance to the jamming transition times the square of the strain. This is the case for example if $\mathbf{y}$ represents a shear deformation, so that $G \sim \Delta z$.

In this scenario, however, the response to compression is an exceptional case. Compression amounts to the special situation that $\mathbf{y}$ is essentially pointing in the same direction as the basis vector $\mathbf{f}^{(1)}$, which we chose to be in the direction of increasing pressure. In this case, all terms in the inner product $\sum_{m} f_{m}^{(1)} y_{m}$ are positive, and of order $\epsilon / \sqrt{N}$, so that $\left\langle\mathbf{f}^{(1)} \mid \mathbf{y}\right\rangle^{2} \sim N \epsilon^{2}$. Now the contribution of the first term in eq. (7) is already extensive by itself, and independent of $\Delta z$. In this picture, the compression response is anomalous since it corresponds to an alignment with a special direction in the force space; in other words, from this point of view too, it is best to think of compression, and not shear, as being the anomalous response!

Conclusion. - Since the jamming transition is about loss of rigidity, it is natural to compare the elasticity of disc packings to rigidity percolation on a random network [8]. From this reference point, what is special about jammed packings is that they have an anomalously large resistance to compression. In addition, the response of packings to compression does not follow the simple scaling relations that govern the particles' relative displacements under shear. This interpretation should be contrasted with the more commonly stated conclusion from comparing to effective medium theory, which would be that the resistance to shear is anomalously small $[2,10]$.

The anomalous behavior of the bulk modulus has a purely geometric origin, as we have shown by numerically extracting it from the dynamical matrix, and interpreting the results in terms of the solution space of the force network ensemble, both of which are purely geometric: It is all a matter of how purely repulsive particles arrange themselves when they are pressed together.

$$
* * *
$$

We thank E. Somfai for providing the numerical code to construct the granular packings, and M. Wyart and V. Vitelli for enlightening discussions. WGE and ZZ acknowledge support from the FOM foundation, and $\mathrm{MvH}$ from the Dutch science foundation NWO through a VIDI grant. WGE thanks the Aspen Center for Physics, where part of this work was done, for its hospitality.

\section{REFERENCES}

[1] Liu A. J. and Nagel S. R., Nature, 396 (1998) 21.

[2] O'Hern C. S., Silbert L. E., Liu A. J. and Nagel S. R., Phys. Rev. E , 68 (2003) 011306.

[3] Silbert L. E., Liu A. J. and Nagel S. R., Phys. Rev. Lett. , 95 (2005) 098301.

[4] Silbert L. E., Liu A. J. and Nagel S. R., Phys. Rev. E , 73 (2006) 041304.

[5] Wyart M., Nagel S. R. and Witten T. A., Europhys. Lett. , 72 (2005) 486.

[6] Wyart M., Silbert L. E., Nagel S. R. and Witten T. A., Phys. Rev. E, 72 (2005) 051306.

[7] Ellenbroek W. G., Somfai E., van Hecke M. and van Saarloos W., Phys. Rev. Lett. , 97 (2006) 258001.

[8] Bolton F. and Weaire D., Phys. Rev. Lett. , 65 (1990) 3449.

[9] Durian D. J., Phys. Rev. E, 55 (1997) 1739.

[10] Makse H. A., Gland N., Johnson D. L. and Schwartz L. M., Phys. Rev. Lett., 83 (1999) 5070.

[11] Somfai E., van Hecke M., Ellenbroek W. G., Shundyak K. and van Saarloos W., Phys. Rev. E, 75 (2007) 020301(R).

[12] Donev A., Cisse I., Sachs D., Variano E. A., Stillinger F. H., Connelly R., Torquato S. and Chaikin P. M., Science, 303 (2004) 990.

[13] Wouterse A., Williams S. R. and Philipse A. P., J. Phys.: Condens. Matter, 19 (2007) 406215.

[14] Mailman M., Schreck C. F., O'Hern C. S. and Chakraborty B., arXiv, 0812.1234 (2008) .

[15] Zeravcic Z., Xu N., Liu A. J., Nagel S. R. and van SAarloos W., arXiv, 0904.1558 (2009).

[16] Moukarzel C. F., Phys. Rev. Lett. , 81 (1998) 1634.

[17] Jacobs D. J. and Thorpe M. F., Phys. Rev. Lett. , 75 (1995) 4051.

[18] Jacobs D. J. and Thorpe M. F., Phys. Rev. E, 53 (1996) 3682.

[19] Mason T. G., Bibette J. and Weitz D. A., Phys. Rev. Lett. , 75 (1995) 2051.

[20] Wyart M., Ann. Phys. Fr. , 30, No. 3 (2005) 1.

[21] WyART M., arXiv , 0806.4653 (2008) .

[22] Somfai E., Roux J.-N., Snoeijer J. H., van Hecke M. and van Saarloos W., Phys. Rev. E, 72 (2005) 021301.

[23] Alexander S., Physics Reports , 296 (1998) 65.

[24] Leonforte F., Tanguy A., Wittmer J. and Barrat J.-L., Phys. Rev. B , 70 (2004) 014203.

[25] Wyart M., Liang H., Kabla A. and Mahadevan L., Phys. Rev. Lett. , 101 (2008) 215501.

[26] Snoeijer J. H., Vlugt T. J. H., van Hecke M. and van Saarloos W., Phys. Rev. Lett. , 92 (2004) 054302.

[27] Snoeijer J. H., Vlugt T. J. H., Ellenbroek W. G., van Hecke M. and van Leeuwen J. M. J., Phys. Rev. E, 70 (2004) 061306.

[28] Snoeijer J. H., Ellenbroek W. G., Vlugt T. J. H. and van Hecke M., Phys. Rev. Lett. , 96 (2006) 098001. 